\title{
Rubella Screening
}

National Cancer Institute

\section{Source}

National Cancer Institute. Rubella Screening. NCI Thesaurus. Code C92873.

A test during pregnancy to determine maternal exposure to the rubella virus that could lead to pregnancy complications or congenital rubella syndrome in the newborn. 